\title{
COL4A1 promotes the growth and metastasis of hepatocellular carcinoma cells by activating FAK-Src signaling
}

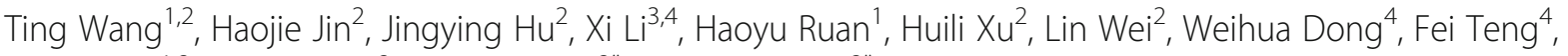
Jianren $\mathrm{Gu}^{1,2}$, Wenxin $\mathrm{Qin}^{2}$, Xiaoying Luo ${ }^{2^{*}}$ and Yujun Hao ${ }^{2^{*}}$

\begin{abstract}
Background: Collagens are the most abundant proteins in extra cellular matrix and important components of tumor microenvironment. Recent studies have showed that aberrant expression of collagens can influence tumor cell behaviors. However, their roles in hepatocellular carcinoma (HCC) are poorly understood.
\end{abstract}

Methods: In this study, we screened all 44 collagen members in HCC using whole transcriptome sequencing data from the public datasets, and collagen type IV alpha1 chain (COL4A1) was identified as most significantly differential expressed gene. Expression of COL4A1 was detected in HCC samples by quantitative real-time polymerase chain reaction (qRT-PCR), western blot and immunohistochemistry (IHC). Finally, functions and potential mechanisms of COL4A1 were explored in HCC progression.

Results: COL4A1 is the most significantly overexpressed collagen gene in HCC. Upregulation of COL4A1 facilitates the proliferation, migration and invasion of HCC cells through FAK-Src signaling. Expression of COL4A1 is upregulated by RUNX1 in HCC. HCC cells with high COL4A1 expression are sensitive to the treatment with FAK or Src inhibitor.

Conclusion: COL4A1 facilitates growth and metastasis in HCC via activation of FAK-Src signaling. High level of COL4A1 may be a potential biomarker for diagnosis and treatment with FAK or Src inhibitor for HCC.

Keywords: COL4A1, HCC, RUNX1, FAK, SrC

\section{Background}

Liver cancer is one of most malignant cancer and causes more than 700,000 deaths per year worldwide [1]. Hepatocellular carcinoma (HCC) is the most common type of liver cancer, accounting for approximately $90 \%$ of all liver cancers [2]. Although surgical resection, orthotopic liver transplantation, radiofrequency thermal ablation and targeted therapy have been applied clinically to liver cancer

\footnotetext{
* Correspondence: luoxy@shsci.org; yjhao@shsci.org

${ }^{2}$ State Key Laboratory of Oncogenes and Related Genes, Shanghai Cancer Institute, Renji Hospital, Shanghai Jiao Tong University School of Medicine, Shanghai 200240, People's Republic of China

Full list of author information is available at the end of the article
}

treatment, the 5-year overall survival rate is still low due to late diagnosis, disease recurrence, metastasis, and the complicated etiology of HCC [3]. Therefore, it is necessary to further understand the molecular mechanisms and identify novel targets for diagnosis as well as treatment of HCC.

Collagens are the most abundant proteins in the human body, accounting for one third of total proteins. In humans, there are at least 28 different types of collagen proteins encoded by 44 collagen genes [4]. They are essential in extra cellular matrix (ECM) which is the major component in tumor microenvironment and can regulate tumor cell behaviors $[5,6]$. Collagen proteins are produced and secreted by fibroblasts [7], osteoblasts [8], 
hematopoietic cells [9], and also can be biosynthesized and regulated by endothelial cells $[10,11]$ and cancer cells [12]. Over the last few years, accumulated evidences have indicated that some collagens are differentially expressed in cancer. Collagen type I contributes to pancreatic, lung, bladder, liver and breast cancer progression [13-17]. Collagen type IV (Col IV) levels are elevated in patients with colorectal liver metastases [18, 19]. Collagen type VI has been identified as biomarker of prognosis for colorectal, ovarian, and pancreatic cancer [20-22]. Collagen type XI is highly expressed in breast cancer, colorectal cancer, and metastatic ovarian carcinoma [23-25]. Collagen type XVII is overexpressed in skin cancers, such as squamous cell carcinoma and melanoma $[26,27]$. However, the functions and mechanisms of collagen genes in HCC are still largely unknown.

Collagen IV is the most abundant constituents of basement membranes of ECM [28]. Col IV is majorly generated and secreted from fibroblasts, endothelial cells, epithelial cells, and some tissue specific cells (e.g. hepatic stellate cells) $[29,30]$. COL4A1 and COL4A2 encode Collagen IV alpha 1 chain and alpha 2 chain, and assemble into $\alpha 1 \alpha 1 \alpha 2$ heterotrimers (Col IV) then secreted into extracellular matrix [31]. COL4A1 mutations have been identified in vascular abnormalities, myopathy, nephropathy, and Walker-Warburg syndrome [32-34]. Upregulated COL4A1 promotes tumor invasion via induction of tumor budding in bladder cancer cells [35]. Overexpressed COL4A1 contributes to the proliferation and migration of breast cancer cells [36]. However, the detailed mechanisms of COL4A1 in HCC progression has not been elucidated.

In this study, expression profiles of collagen genes were comprehensively screened and COL4A1 was identified as the most abundant and significantly differential expressed collagen gene in HCC. Upregulation of COL4A1 promoted the proliferation and metastasis of HCC cells through FAK-Src signaling. We first found that expression of COL4A1 is upregulated by transcriptional factor RUNX1. Moreover, we innovatively discovered that HCC cells with high COL4A1 expression were sensitive to the treatment of FAK or Src inhibitor. Our findings suggest that the upregulation of COL4A1 is important in the progress of $\mathrm{HCC}$, and COL4A1 maybe a potential target for diagnosis and treatment of HCC.

\section{Materials and methods}

\section{Clinical specimens and TCGA data analysis}

89 pairs of HCC tissues and peritumor samples for quantitative real-time polymerase chain reaction (qRTPCR) analyses, 10 pairs of HCC tissues and peritumor samples for western blot analyses and 4 pairs of paraffinembedded tissue for immunohistochemistry (IHC) analyses were obtained from patients at Zhongshan Hospital of Fudan University (Shanghai, China) from 2004 to
2005. Ethical approval was examined and certified by the Ethics Committee of Zhongshan Hospital Biomedical Research Department, and written informed consent was obtained from all involved patients. The slides of paraffin-embedded HCC tissues and paired peritumor samples for IHC analyses were purchased from Liaoding (Shanghai, China) $(n=20)$. The whole transcriptome sequencing (RNA-seq) data of 374 liver tumor tissues and 50 adjacent non-tumor tissues were obtained from The Cancer Genome Atlas (TCGA) liver cancer dataset (LIHC) (http://cancergenome.nih.gov). mRNA level of COL4A1 of 20 tumor types and another 4 datasets of HCC were obtained from the Oncomine database (https://www.oncomine.org/resource/main.html). Representative IHC staining results of COL4A1 in HCC and normal liver tissues were obtained from the Human Protein Atlas online database (https://www.proteinatlas.org, magnification, $\times 40$ ).

\section{Cell lines and culture}

Human HCC cell lines (HepG2, PLC/PRF/5, Hep3B, and SK-Hep1) were purchased from the American Type Culture Collection (Manassas, VA, USA). HCC cell line (Huh7) was provided by Riken Cell Bank (Tsukuba, Japan). Human normal liver cell line (L02) and HCC cell line (SMMC7721) were purchased from Cell Bank of the Chinese Academy of Sciences (Shanghai, China). All cell lines were cultured in Dulbecco's modified Eagle's medium (DMEM; Gibco, USA) supplemented with 10\% fetal bovine serum (FBS; Gibco, USA), 100 units $/ \mathrm{ml}$ penicillin and $100 \mu \mathrm{g} / \mathrm{ml}$ streptomycin (Gibco, USA), and incubated at $37{ }^{\circ} \mathrm{C}$ in a humidified incubator containing 5\% CO2.

\section{Antibodies and plasmids}

Antibodies used in this study were shown in Additional file 1: Table S1. Human expression vectors for HA-RUNX1, Myc-RUNX2 and Myc-RUNX3 recombinant proteins were generated using PCMV backbone vector. The promoter of COL4A1 was cloned into pGL3 basic vector. The subcloning primers were listed in Additional file 2: Table S2.

\section{Western blot}

Cells were washed three times with cold phosphate buffered saline (PBS) and total cellular protein was extracted using RIPA lysis buffer (Qiagen, Germany) supplied with proteinase inhibitor cocktail and phosphatase inhibitor (Roche Applied Science, Switzerland). The lysates were incubated on ice for $30 \mathrm{~min}$ followed by centrifugation at $4{ }^{\circ} \mathrm{C} 12000 \times \mathrm{g}$ for $30 \mathrm{~min}$. Protein concentrations were analyzed using the Bicinchoninic Acid (BCA) Kit (Pierce, Rockford, IL). $40 \mu \mathrm{g}$ of total proteins were separated by $10 \%$ sodium dodecyl sulfate-polyacrylamide gel 
electrophoresis (SDS-PAGE) and transferred onto $0.22 \mu \mathrm{m}$ polyvinylidene fluoride membrane (PDVF; Millipore). The membranes were blocked with $5 \%$ non-fat dried milk for an hour at room temperature, and then incubated with primary antibodies overnight at $4{ }^{\circ} \mathrm{C}$. Protein bands were visualized by the enhanced chemiluminescence (ECL) detection kit (Tanon, China). Quantification of western blots were analyzed by Image J.

\section{Immunohistochemistry (IHC)}

Paraffin-embedded HCC tissues and peritumor samples were completely deparaffinized, and then performed antigen retrieval using antigen retrieval solution (Beyotime, Shanghai, China). Immunohistochemistry staining were applied with Immunohistochemistry Application Solutions Kit (Cell Signaling Technology, Dancers, USA) according to the manufacturer's instructions. Briefly, the slides were incubated with primary anti-COL4A1 (1:200, Abcam) overnight at $4{ }^{\circ} \mathrm{C}$ and incubated the secondary antibody $30 \mathrm{~min}$ at room temperature. Then, the slides were counter stained with hematoxylin for $3 \mathrm{~min}$. Staining results were independently evaluated by two experienced pathologists who were blinded to all clinical data.

\section{RNA extraction and qRT-PCR}

Total RNA was extracted from cell lines and tissue samples using the TRIzol kit (Invitrogen, Carlsbad, CA, USA). RNA $(1 \mu \mathrm{g})$ was reverse-transcribed into cDNA immediately using Prime-Script RT kit (Takara, Shiga, Japan) following manufacturer's instructions. qRT-PCR was carried out with SYBR Premix EX Tag (Takara) on an ABI Prism 7500 fast RT-PCR instrument (Applied Biosystems, Foster City, CA). Each experiment was performed in triplicate. $\beta$-actin was used as the internal reference gene. Data were acquired during the extension step. Objective CT values were normalized to $\beta$-actin and $2^{-\Delta \mathrm{Ct}}$ method was used to calculate relative mRNA levels of gene expression. Primer sequences for qRTPCR were listed in Additional file 2: Table S2. The qRTPCR primers for COL4A1 are not exon spanning type, but their specificity has been tested.

\section{Lentiviral constructs and cell infection}

To knockdown COL4A1 in cell lines, two independent shRNA sequences were designed and cloned into the pGreen-Puro vector (System Biosciences, CA). Another shRNA with a non-targeting sequence was used as a negative control (NC). The shRNA sequences were listed in Additional file 2: Table S2. Virus packaging was performed in HEK $293 \mathrm{~T}$ cells after co-transfection of pGreenPuro-shCOL4A1 with packaging plasmid pPACK-GAG, pPACK-REV (System Biosciences) and envelope plasmid pVSV-G (System Biosciences) using
Lipofectamine 3000 (Invitrogen, Carlsbad, CA, USA). Viruses were harvested $48 \mathrm{~h}$ after transfection. Medium with viral supernatant was filtered through a $0.45 \mu \mathrm{m}$ strainer and viral titers were determined. SMMC7721 cells and SK-Hep1 cells were infected with lentivirus using polybrene $(6 \mu \mathrm{g} / \mathrm{ml}$, Sigma).

CRISPR/Cas9 Synergistic Activation Mediator (SAM) is an engineered protein complex, which is a powerful tool for strong transcriptional activation of endogenous genes at targeted sites. Complete SAM system consists of two separate lentiviral vectors: the dCas9-VP64-puro vector and the sgRNA (COL4A1)-MS2-P65-HSF1-G418 (single guiding RNA) vector. Plasmids design and two lentiviruses packaging were done at Genechem Co., Ltd. (Shanghai, China). sgRNAs with matching COL4A1 gene promoter sequences (Gene Bank ID: NM_001845) were listed in Additional file 2: Table S2. HepG2 cells and $\mathrm{PLC} / \mathrm{PRF} / 5$ cells were infected with the lentivirus of dCas9-VP64-puro. After two weeks of puromycin $(1 \mu \mathrm{g} /$ $\mathrm{ml})$ selection, cells were infected with indicated sgRNA (COL4A1)-MS2-P65-HSF1-G418 lentivirus and selected with G418 $(600 \mu \mathrm{g} / \mathrm{ml})$.

\section{siRNA knockdown}

The siRNAs targeting human COL4A2, COL3A1, COL1A1, RUNX1 and the scramble siRNA control were obtained from Biotend (Shanghai, China). siRNAs were transfected into indicated cells with Lipofectamine 3000 according to manufacturer's instructions. Cells were harvested $48-72 \mathrm{~h}$ post transfection for various assays. Sequences for siRNA were listed in Additional file 2: Table S2.

\section{Proliferation, migration and invasion analysis}

Cell proliferation, invasion and migration assays were measured with the xCELLigence System's Real-Time Cell Analyzer (RTCA, Roche/ACEA Biosciences) placed in a humidified incubator and maintained at $37^{\circ} \mathrm{C}$ with $95 \%$ air/ $5 \% \mathrm{CO} 2$. This system continuously monitored electrical impedance which created by cell adhesion and proliferation in microelectrode-integrated membrane, and outputted as a unit-less parameter (cell index). For proliferation assays, $1 \times$ $10^{4}$ to $3 \times 10^{4}$ cells were seeded into E-plate 16 (ACEA Biosciences) with $200 \mu \mathrm{L}$ DMEM containing $10 \%$ FBS $(n=3)$. Cell index was normalized to baseline reading at time point 0 , and measured every $30 \mathrm{~min}$ for $72 \mathrm{~h}$. Migration and invasion assays were performed in 16-well CIM plates (ACEA Biosciences). For migration assays, $1.5 \times 10^{5}$ cells were seeded as triplicates in the upper chamber in serum free medium. Upper chamber was then placed on the lower part of the CIM-device containing DMEM with 10\% FBS as a chemoattractant. Cell index was measured every $30 \mathrm{~min}$ for $48 \mathrm{~h}$. For invasion assays, upper chamber of CIM-16 plate was initially coated with Matrigel (BD Biosciences, Bedford, 
MA, USA) diluted in serum free medium at a ratio of 1:20. Then, next steps were same with migration assays.

For cell proliferation assay, Cell Counting Kit-8 (Dojindo, Japan) was also applied according to the manufacturer's instructions. Briefly, $3 \times 10^{3}$ cells per well were planted in a 96-well plate. Absorbance at OD450 was measured for 4 consecutive days and used to plot cell growth curves. For colony formation assay, $8 \times 10^{2}$ cells were seeded in 6-well plates and maintained in DMEM medium with 5\% FBS. After 14 days, cells were washed with $\mathrm{PBS}$ and stained with $0.5 \%$ crystal violet. For transwell assay, $5 \times 10^{4}$ cells were seeded into upper chambers (transwells with $8-\mu \mathrm{m}$ pores, Corning, USA) without FBS, and DMEM containing 10\% FBS was introduced to the lower chambers (24-well plates). After 2 days, cells remaining on the upper surface of the filter were removed using a cotton swab with PBS, then transwells were fixed and stained with $0.5 \%$ crystal violet. The migratory cells were photographed and counted in 5 different fields per well.

\section{Drug treatment}

FAK inhibitor Defactinib (VS-6063) was purchased from CSNpharm (CSN17445, Shanghai, China). Src inhibitor Saracatinib (AZD0530) was purchased from Selleck Chemical (S1006, Huston, TX, USA). To evaluate inhibitory activity of FAK or Src inhibitor (Defactinib or Saracatinib), cells were firstly seeded at a density of $5 \times 10^{3}$ in 96-well plates and incubated overnight. Then Defactinib or Saracatinib was added at indicated concentrations. After 2 days, CCK8 was applied to measure survival cells following manufacturer instructions.

\section{Wound healing assay}

Cells were grown in 12-well plates at $95 \%$ confluency. A linear wound was scratched with a $200 \mu \mathrm{L}$ sterile pipette tip across the monolayers. After washing with PBS to remove cell debris, adherent cells were incubated in medium with $10 \%$ FBS. Wounded monolayers were photographed every $3 \mathrm{~h}$ for $24 \mathrm{~h}$.

\section{Tumor xenograft models}

Subcutaneous xenograft mouse model was used to assess tumor growth. Animal experiments were approved by the Ethics Committee of the Renji Hospital, Shanghai Jiao Tong University School of Medicine. Female nude mice (age, 4-5 weeks; weight, 14-16 g; Institute of Zoology, Chinese Academy of Sciences) were randomly divided into three groups: two COL4A1 knockdown groups and one NC group ( $n=7$ per group). A total of $2 \times 10^{6}$ SMMC7721 cells in $100 \mu \mathrm{L}$ of DMEM without FBS were injected into right axillary fossa of nude mice. Tumor volume was measured by caliper measurements every 3 days and calculated with the formula of (length $x$ width $\left.^{\wedge}\right) / 2$.

\section{Statistical analysis}

Data were analyzed using GraphPad Prism 7. Results were presented as mean \pm standard deviation (SD, $n=3)$. Statistical differences between groups were evaluated by the student's $t$ test (paired/unpaired). Pearson correlation tests were performed on correlation analyses. Two-way analysis of variance (ANOVA) followed by Tukey's multiple comparisons test was performed to compare significant difference and calculate the $P$-value between the different groups. It was considered as statistically different when $P<0.05$ ( ${ }^{*} P<0.05,{ }^{* * *} P<0.01$, ${ }^{* * * *} P<0.001$, $\left.{ }^{* * * * * *} P<0.0001\right)$, otherwise not significant (ns).

\section{Results}

COL4A1 is upregulated in HCC

To identify the cancer-related collagen genes in HCC, we first analyzed expression level for all 44 members of collagen genes in HCC using RNA-seq data from TCGA-LIHC dataset. 31 of 44 (70.5\%) collagen genes were differentially expressed in 374 liver cancer samples compared with 50 normal liver samples. Among 27 upregulated genes and 4 downregulated genes, COL1A1, COL1A2, COL4A1, and COL4A2 were significantly upregulated genes (Fig. 1a and Additional file 3: Table S3). In 50 paired $\mathrm{HCC}$ and normal liver samples from TCGA dataset, COL1A1 and COL1A2 were only upregulated in $74 \%$ (37 of 50) of HCC samples, but COL4A1 and COL4A2 were upregulated in $100 \%$ (50 of 50) of HCC samples (Additional file 4: Figure S1A). Furthermore, COL4A1 was the most significantly upregulated collagen gene in HCC in term of average fold changes (cancerous tissues/noncancerous liver tissues, 1.69 for COL1A1; 1.69 for COL1A2; 2.10 for COL4A1 and 1.92 for COL4A2). This result was confirmed by other four HCC datasets (Roessler Liver Statistics, Roessler Liver 2 Statistics, Wurmbach Liver Statistics, and Mas Liver Statistics) in Oncomine database (Fig. 1b). This result was also validated in our own dataset which we submitted previously (GSE84402) [37], and COL4A1 was upregulated in 11 of 14 HCC samples (Additional file 4: Figure S1B). In another 89 pairs of HCC and adjacent normal tissues, COL4A1 was upregulated in 79 of 89 HCC samples (88.7\%) by qRT-PCR analysis (Fig. 1c and Additional file 4: Figure S1C). In addition, COL4A1 was upregulated not only in HCC but also in most types of cancer, including colorectal cancer, gastric cancer as well as head and neck cancer, etc. (Additional file 4: Figure S1D).

Since mRNA levels of genes are not always consistent with their protein levels, protein level of COL4A1 in clinical HCC tissues needs to be further analyzed. Firstly, COL4A1 was highly expressed in all HCC samples (10 


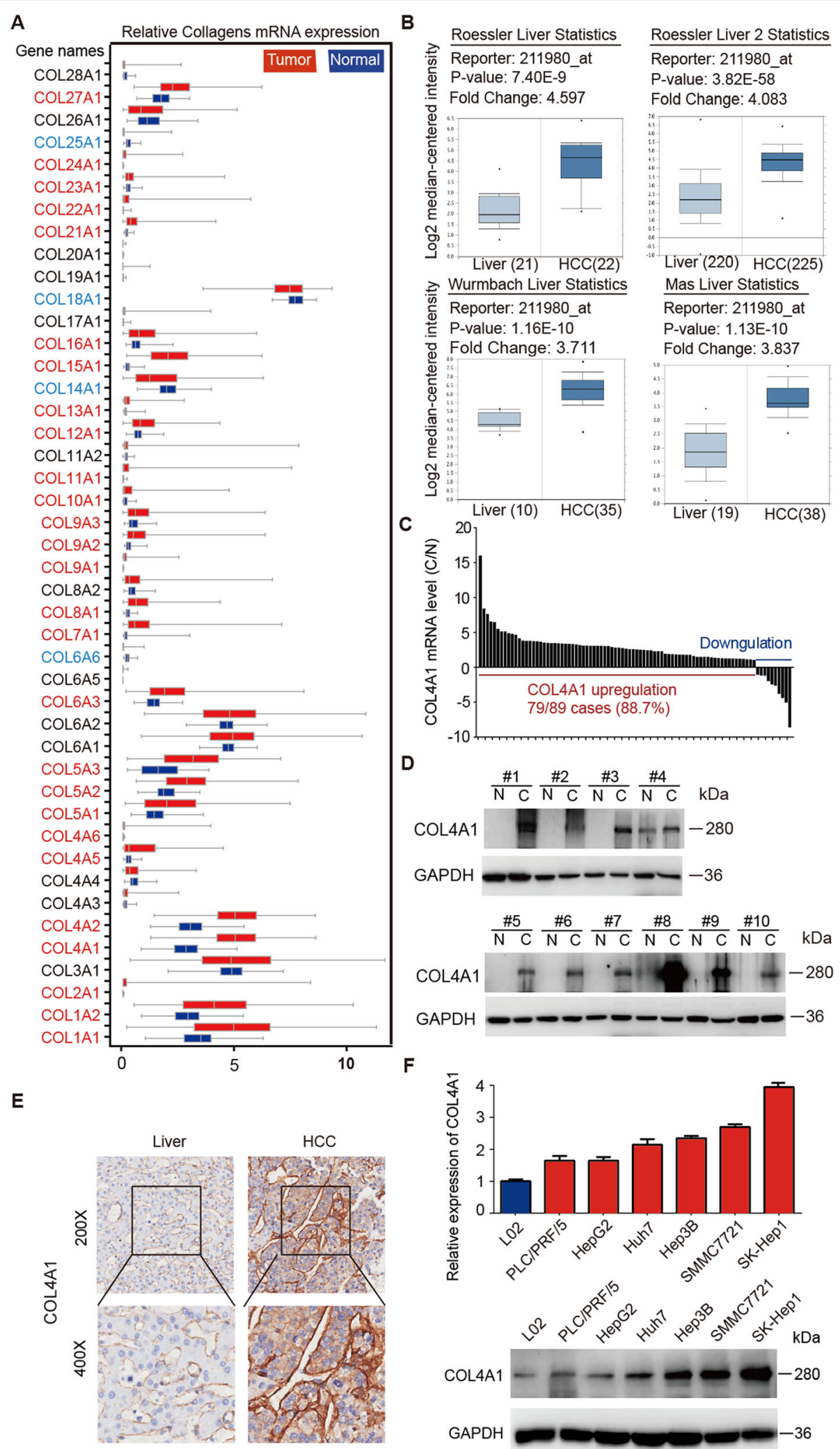

Fig. 1 (See legend on next page.) 
(See figure on previous page.)

Fig. 1 Upregulation of COL4A1 in HCC. a Box-whisker Plot indicated the mRNA expression profiles of 44 collagen genes in TCGA dataset. The gene name shown in red and blue illustrates the 27 upregulation genes and 4 downregulation genes in tumor tissues compared with normal liver tissues, respectively. $\mathbf{b}$ Expression of COL4A1 was significantly upregulated in HCC compared with normal liver tissues in 4 datasets, including Roessler Liver Statistics, Roessler Liver 2 Statistics, Wurmbach Liver Statistics, and Mas Liver Statistics. c mRNA levels of COL4A1 in paired HCC samples were detected by qRT-PCR $(n=89)$. Fold changes $(C / N)$ were presented. C, cancerous tissues; $N$, noncancerous liver tissues. $\mathbf{d}$ The protein levels of COL4A1 in 10 paired cancerous tissues $(C)$ and the matched adjacent noncancerous liver tissues $(\mathrm{N})$ from HCC patients were analyzed by western blot. e COL4A1 proteins were highly expressed in HCC tissues. Immunohistochemistry staining of COL4A1 was performed in paired HCC samples and normal liver tissues $(n=24)$. The representative images were shown. $\mathbf{f}$ Expression levels of COL4A1 were measured by qRT-PCR and western blot in indicated HCC cell lines

of 10) compared with normal liver tissues by western blot analyses (Fig. 1d). Secondly, we performed IHC staining of COL4A1 in clinical specimens, and COL4A1 was highly expressed in 17 of $24(70.8 \%)$ human HCC specimens compared with normal liver tissues (Fig. 1e). We also analyzed the data of IHC staining in the Human Protein Atlas database, consistently, moderate COL4A1 staining was observed in HCC samples and no obvious staining of COL4A1 was found in hepatocytes (Additional file 4: Figure S1E).

We next examined the mRNA and protein levels of COL4A1 in six HCC cell lines (HepG2, Hep3B, SKHep1, SMMC7721, Huh7, and PLC/PRF/5) and an immortalized liver cell line L02. As shown in Fig. 1f, both mRNA and protein levels of COL4A1 were higher in HCC cell lines than that in L02. Since Col IV is normally secreted to extracellular matrix to perform its function [38], we detected the secretion of COL4A1 in medium supernatant of $\mathrm{HCC}$ cell lines. As shown in Additional file 4: Figure S1F, more COL4A1 was secreted into culture medium of SK-Hep1 and SMMC7721 cells which expressed high levels of COL4A1, but low levels of COL4A1 in culture medium of HepG2 and PLC/PRF/5 cells which expressed relative low levels of COL4A1, indicating that cells with high expression of COL4A1 will secrete more COL4A1 into the extracellular matrix.

COL4A1 and COL4A2 are only $127 \mathrm{bp}$ apart on chromosome 13q34 in a head-to-head arrangement and share a bidirectional promoter. COL4A2 expression is significantly positively correlated with COL4A1 [31, 39]. COL1A1 has been reported to overexpress in HCC samples [40]. But the expression of COL3A1 is similar in HCC samples compared with non-tumor liver tissues (Fig. 1a). So, we checked the mRNA and protein levels of COL4A2, COL1A1 and COL3A1 in HCC cell lines as positive or negative controls. As shown in Additional file 4: Figure S1G\&H, both mRNA and protein levels of COL4A2 were higher in HCC cell lines than that in L02, which was consistent with the trend of COL4A1. Although COL1A1 was highly expressed in HCC samples (Fig. 1a), the expression of COL1A1 in HCC cell lines was not more than L02 cells (Additional file 4: Figure S1G\&H), suggesting that other cell types in tumor microenvironment might contribute more for COL1A1 expression than HCC cells. The expression of COL3A1 had no difference in HCC cell lines compared with L02 (Additional file 4: Figure S1G\&H).

We analyzed correlation between COL4A1 expression and clinicopathological parameters of HCC. Although not obvious, statistically, the expression of COL4A1 were slightly higher in higher stages of HCC (III and IV) than that in lower stages (I and II) (Additional file 4: Figure S1I). Together, our results indicate that COL4A1 is most significantly upregulated collagen gene in HCC cells and may plays critical roles in HCC progression.

\section{COL4A 1 promotes proliferation, migration and invasion of HCC cells}

According to the expression patterns of COL4A1 in HCC cell lines, we knocked down COL4A1 in SMMC7721, SKHep1, HepG2 and PLC/PRF/5 cells by two independent shRNAs (Fig. 2a and Additional file 5: Figure S2A). Knockdown of COL4A1 significantly inhibited the proliferation (Fig. 2b and Additional file 5: Figure S2B), migration (Fig. 2c and Additional file 5: Figure S2C) and invasion (Fig. 2d and Additional file 5: Figure S2D) in those cells by real-time cell analyzer (RTCA). These data were validated by wound healing assay and colony formation assay (Fig. 2e \& f). Moreover, we conducted subcutaneous xenograft tumor experiments in nude mice, and results showed that knockdown of COL4A1 significantly reduced the growth of subcutaneous xenograft tumors derived from SMMC7721 cells (Fig. 2g).

We next overexpressed COL4A1 in HepG2 cells and PLC cells by CRISPR/Cas9 synergistic activation mediator (SAM) strategy (Fig. 3a). Overexpression of COL4A1 promoted the abilities of cell proliferation, migration and invasion (Fig. 3b-d).

Given that COL4A1 normally perform its function through assembling as heterotrimers with COL4A2, we explored whether COL4A2 would have similar biological function as COL4A1. As shown in Additional file 6: Figure S3, knockdown of COL4A2 in SMM7721 and SK-Hep1 by siRNA could inhibit the proliferation and migration of those cells. In addition, COL1A1 has been shown to promote HCC progression [17, 40,41]. We also found that knockdown of COL1A1 inhibited the proliferation and migration of SMM7721 and SK-Hep1 


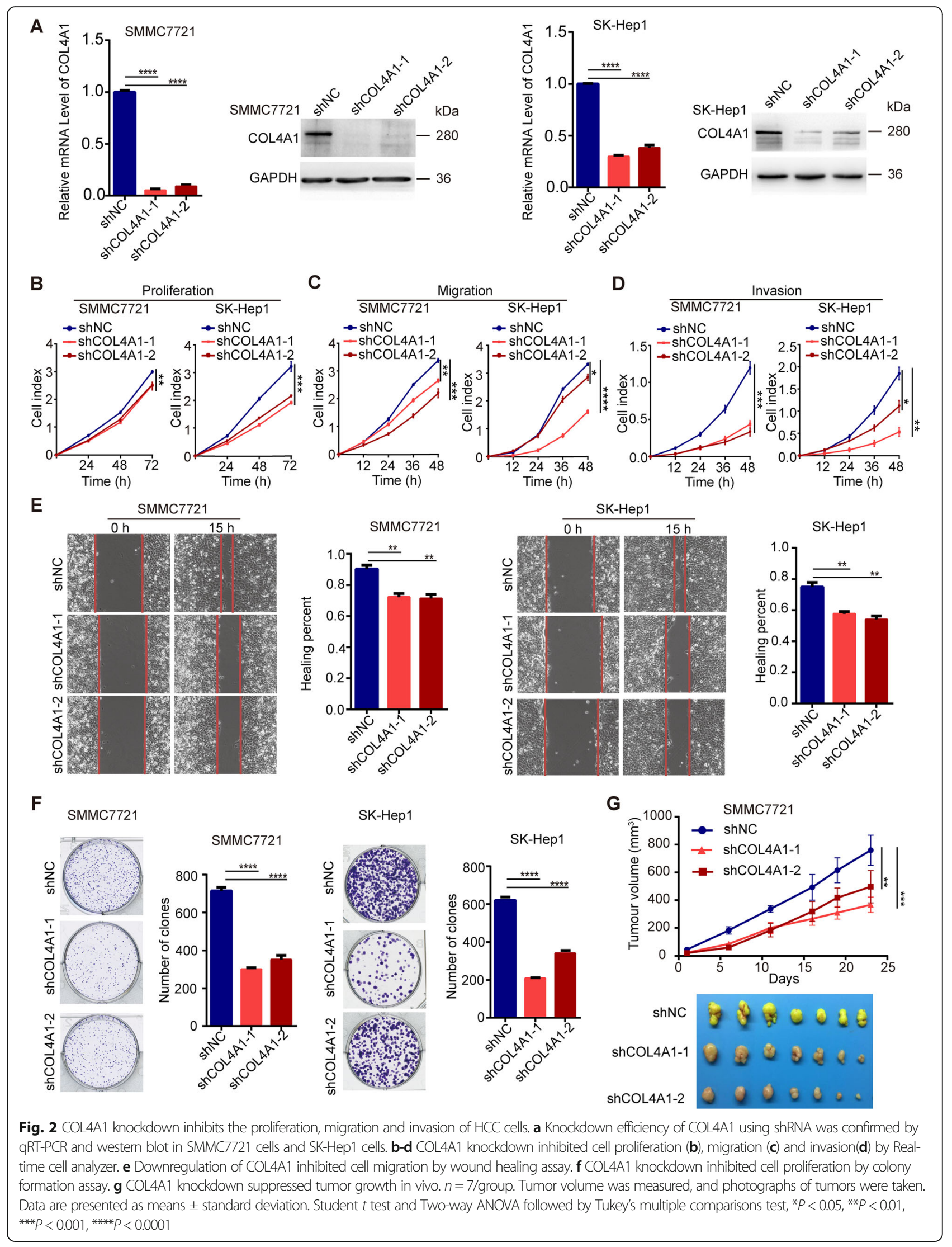




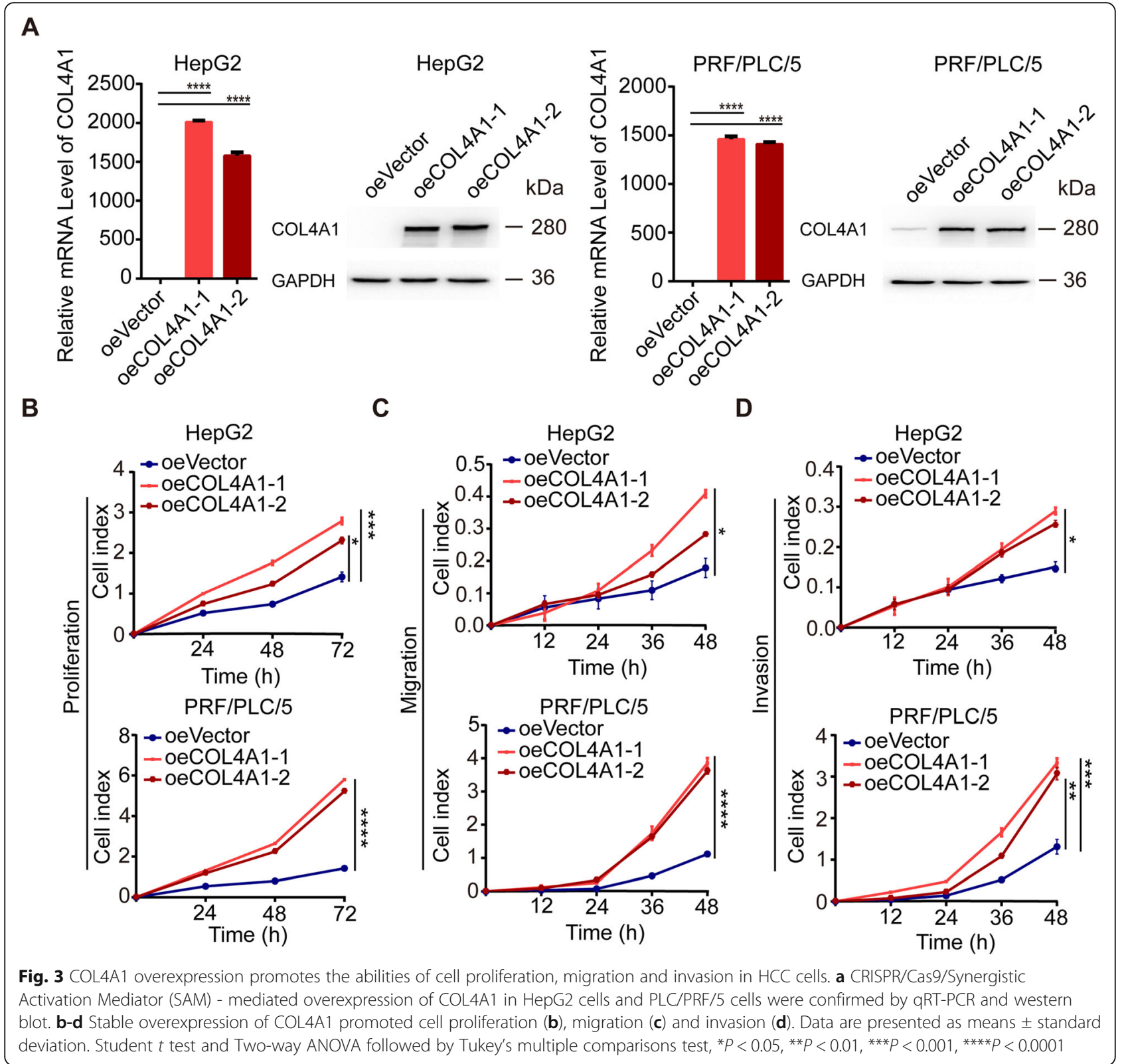

cells (Additional file 7: Figure S4). However, knockdown of COL3A1 had no impact on HCC cells proliferation and migration (Additional file 8: Figure S5). Taken together, collagen IV in liver cancer cells could promote HCC progression.

\section{COL4A1 is transcriptionally activated by RUNX1}

It has been described that RUNXs (Runt-related transcription factor family) are transcriptional factors of collagen genes [42-44]. To study whether COL4A1 is regulated by RUNXs in HCC, we screened the correlation between COL4A1 and RUNX1, RUNX2 or RUNX3 in HCC. As shown in Fig. 4a and Additional file 9: Figure S6A, the expression of COL4A1 was most positively correlated with RUNX1 $(r=0.5800, P<0.0001)$. In addition, similar to COL4A1 expression, RUNX1 was highly expressed in HCC tissues, but expression levels of RUNX2 and RUNX3 were not significantly different between HCC tissues and normal liver tissues (Fig. $4 \mathrm{~b}$ and Additional file 9: Figure S6B). We further found that overexpression of RUNX1, but not RUNX2 or RUNX3 dramatically elevated COL4A1 expression (Fig. 4c and Additional file 9: Figure S6C\&D). In addition, knockdown of RUNX1 significantly decreased expression of COL4A1 in SMMC7721 cells and SK-Hep1 cells (Fig. 4d). Next, the promoter of COL4A1 was cloned into pGL3 basic vector. As shown in Fig. 4e, overexpression of RUNX1 activated the transcription of COL4A1 in 


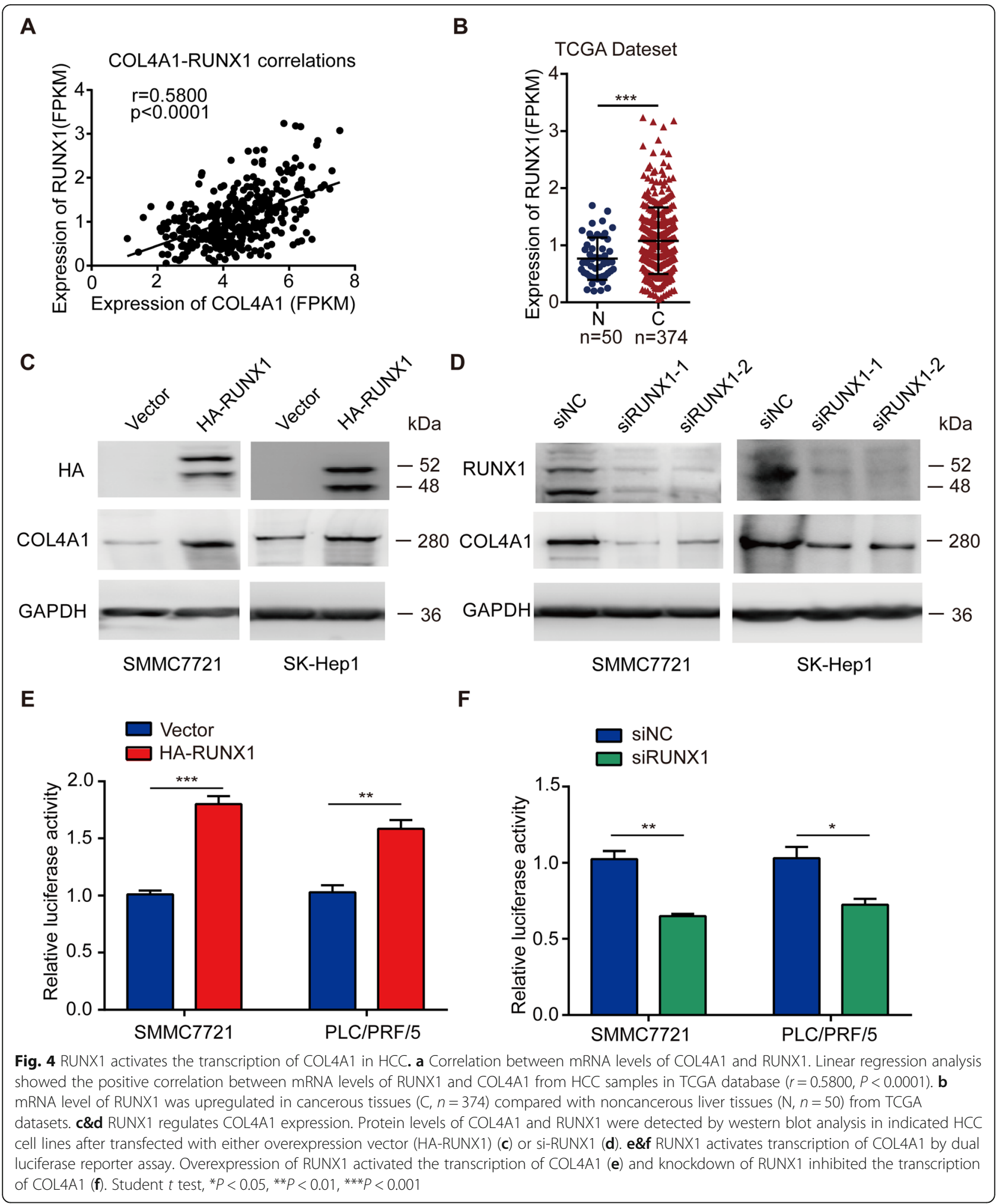

SMMC7721 cells and PLC cells with dual-luciferase assay system. Knockdown of RUNX1 inhibited the transcription of COL4A1 (Fig. 4f). Several putative binding sites of RUNX1 locating in COL4A1 promoter region were found by an online website JASPAR (http://jaspar. genereg.net/) (Additional file 9: Figure S6E). We further analyzed correlation between RUNX1 expression and clinical stages of HCC. Similar with COL4A1, the 
expression of RUNX1 were slightly higher in high stages of HCC than that in low stages statistically (I/II vs III/ IV, $P<0.05$ ) (Additional file 9: Figure S6F). These data suggest that RUNX1 is a transcriptional factor of COL4A1 and probably is one of reasons for upregulation of COL4A1 in HCC.

\section{Overexpression of COL4A1 activates the FAK-Src signaling} It has been reported that collagen transduces signals through binding cell surface integrin or non-integrin receptors [45]. The major receptors for Col IV are integrins such as integrin $\alpha 1$ (ITGA1) and integrin $\beta 1$ (ITGB1) [46-48]. We analyzed the expression of ITGA1 and ITGB1 in 50 paired HCC samples and normal liver tissues from TCGA dataset. Similar expression levels of ITGA1 was detected in HCC and normal liver tissues, whereas expression of ITGB1 was slightly higher in HCC samples compared with normal liver tissues (Additional file 10: Figure S7A). And protein levels of ITGA1 were similar in HCC cell lines compared with L02 cells (Additional file 10: Figure S7B). The binding of collagen to integrin led to the activation of downstream signaling pathways. FAK is one of the major substrates of integrin and it can further phosphorylate and activate downstream signaling molecules including $\mathrm{Src}$ and AKT [49-52]. Several studies have showed that aberrant expression of collagens are associated with phosphorylation of ERK1/2 and STAT3 as well as the expression of metalloproteinase-9 (MMP-9), $\beta$-catenin and E-cadherin [46, 53-55].

To investigate the downstream signaling of COL4A1 in HCC, we detected the levels of phosphorylation and expression for those downstream proteins in parental cells and COL4A1 overexpression or knockdown cells. We observed that overexpression of COL4A1 increased phosphorylation levels of FAK, Src, and AKT (Fig. 5a). Knockdown of COL4A1 significantly reduced phosphorylation levels of them (Fig. 5b). Whereas, knockdown of COL4A1 had no effect on phosphorylation levels of STAT3 and ERK1/2 as well as protein levels of MMP9, $\beta$-catenin, and E-cadherin (Additional file 10: Figure S7C). Moreover, knockdown of COL4A2 decreased phosphorylation levels of FAK and Src like COL4A1 (Additional file 10: Figure S7D). However, knockdown of COL1A1 and COL3A1 had no effect on FAK activity (Additional file 10: Figure S7E\&F). Together, type IV collagen promotes HCC progression by activating FAKSrc signaling.

Inhibitors of FAK and Src selectively suppress the growth of HCC cells with high expression of COL4A1

Based on promotion effects of upregulation of COL4A1 in tumor growth through activating FAK-Src signaling in HCC cells, we wonder if FAK or Src inhibitor could effectively suppress the growth of HCC cells with high expression of COL4A1. We selected Defactinib (FAK inhibitor) and Saracatinib (Src inhibitor) to evaluate their efficacy on HCC cells, because several clinical trials of these two inhibitors for cancer treatment are underway (https://clinicaltrials.gov). Results showed that both Defactinib and Saracatinib could significantly inhibit the growth of HCC cells with high expression of COL4A1 (SK-Hep1, Hep3B, and SMMC7721) (Fig. 6a), while they had little effect on inhibiting the growth of HCC cells with low expression of COL4A1 (PLC/PRF/5, HepG2, and Huh7) (Fig. 6b). To further verify whether cell sensitivity to FAK or Src inhibitors was associated with COL4A1 expression, we examined cell sensitivity to those two inhibitors after modulating COL4A1 expression in HCC cells. As expected, depletion of COL4A1 in HCC cells with high COL4A1 expression reduced their sensitive to Defactinib or Saracatinib treatment (Fig. 6c\&d). Whereas, overexpressing COL4A1 in HCC cells with low COL4A1 expression could increase their sensitive to Defactinib or Saracatinib treatment (Fig. 6e\&f). Therefore, COL4A1 may be a biomarker for treatment with FAK or Src inhibitor for HCC patients.

\section{Discussion}

In this study, we first put forward the role of COL4A1 in HCC tumorigenesis. COL4A1 is dramatically upregulated collagen gene in $\mathrm{HCC}$ by screening the expression patterns of all 44 collagen genes in liver cancer from the TCGA-LIHC database. COL4A1 promotes the growth and metastasis of $\mathrm{HCC}$ cells by activating FAK-Src signaling. RUNX1 is a transcriptional factor of COL4A1 and activates the expression of COL4A1 in HCC. Targeting FAK or Src may be an effective strategy to treat HCC patients with high expression of COL4A1 (Fig. 7).

Collagen proteins form the scaffold of tumor microenvironment and are important for tumor infiltration, angiogenesis, and metastasis [5]. Some collagen genes have been found aberrant expression during carcinogenesis in various types of cancer. However, only a few studies on the expression and function of collagen genes have been reported in HCC. Some studies reported that COL1A1 was upregulated in HCC and could promote HCC progression [17, 40, 41]. Based on bioinformatics analysis, Liu et al. reported that COL4A1 and COL4A2 were significantly correlated with hepatocarcinogenesis and HCC progression [56]. In this study, we analyzed the expression patterns of all 44 collagen genes in liver cancer from TCGA-LIHC database, and found that the expression of around $70 \%$ collagen genes are dysregulated. Among these dysregulated collagen genes, expression of COL4A1 is most abundant and significantly upregulated in HCC. 
A
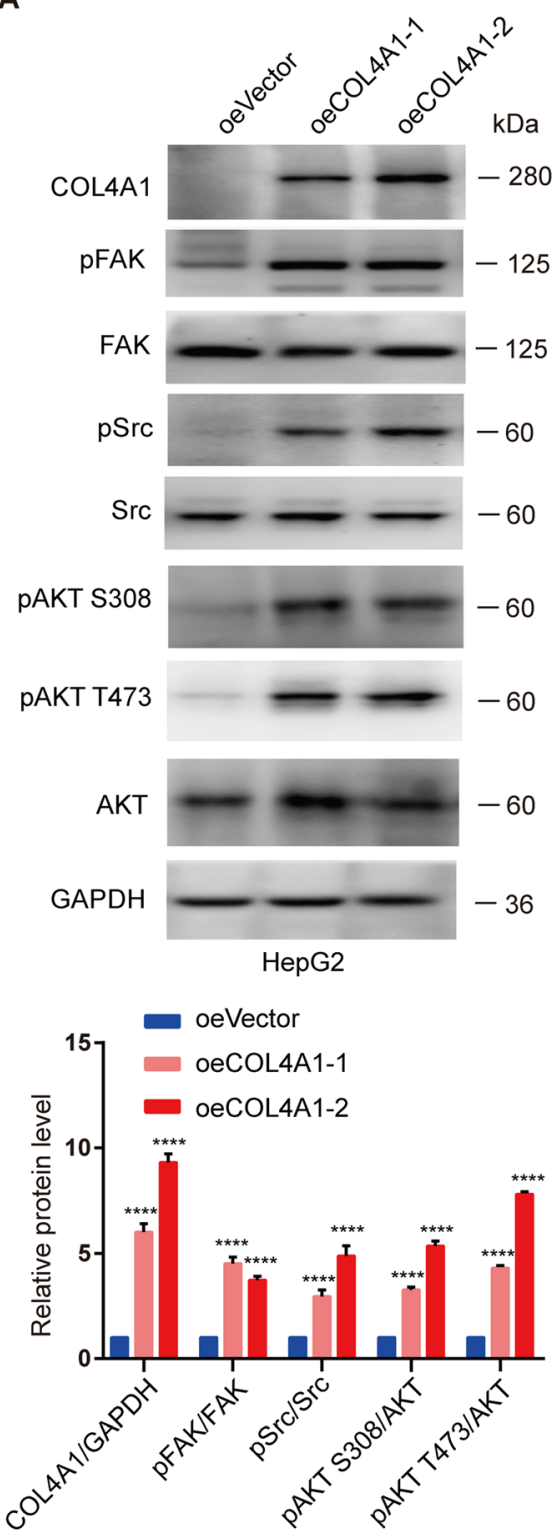

B

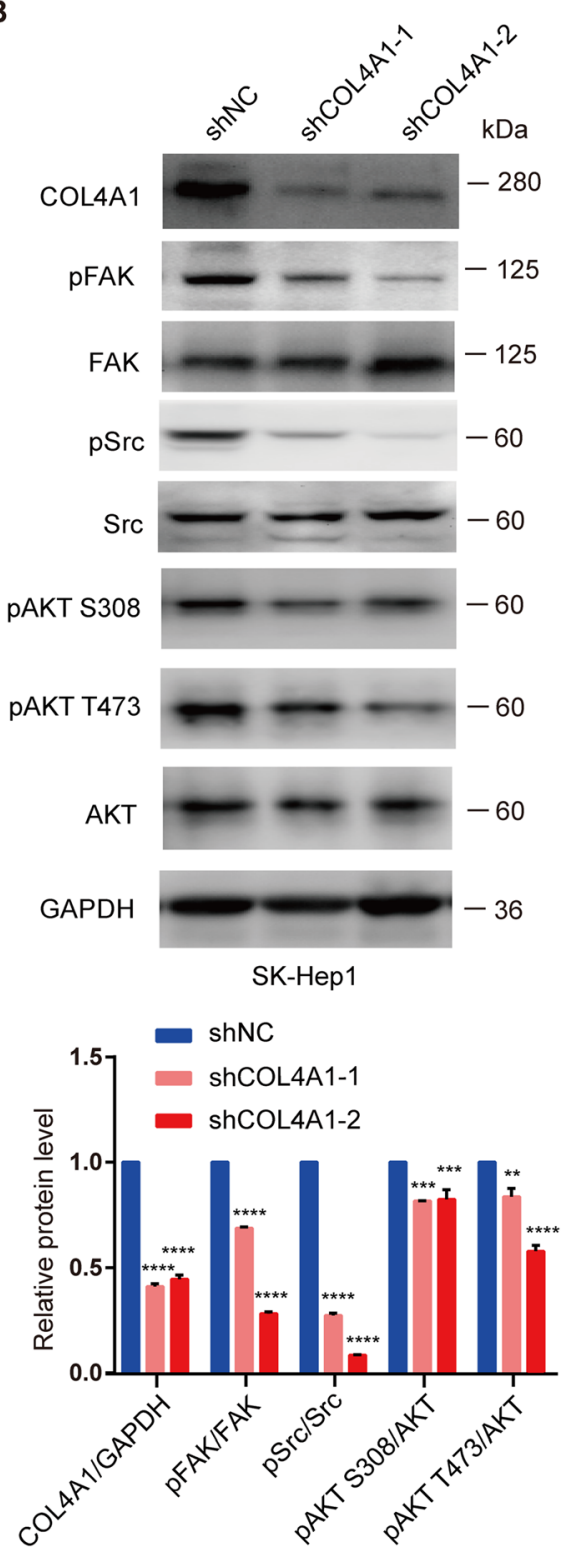

Fig. 5 Overexpression of COL4A1 activates FAK-Src signaling. a Phosphorylation of FAK, Src, and AKT were analyzed by western blot in COL4A1 overexpressed HCC cells. b Phosphorylation of FAK, Src, and AKT were analyzed by western blot in COL4A1 knockdown HCC cells. Quantification of western blots were analyzed by Image J. Student $t$ test, ${ }^{* *} P<0.01$, ${ }^{* *} P<0.001,{ }^{* * *} P<0.0001$

Although Col IV has been reported to associate with the progression of cancer $[36,46]$, the detail molecular mechanisms are not well documented. Burnier et al. showed that Col IV activated FAK in liver metastasis sites generated by different primary tumors [57]. Our data showed COL4A1 expression could affect the phosphorylation of FAK in HCC cells, suggesting that COL4A1 activates FAK signaling to promote HCC progression. Chen et al. showed that COL4A1 regulated tumor cell stiffness and migration through activation of Src and ERK1/2 [46]. Espinosa et al. reported that Col
IV increased the expression and activation of ERK $1 / 2$ [53]. In breast cancer, COL4A1 induced MMP-9 expression by activating Src phosphorylation [54]. In our study, COL4A1 overexpression increased the phosphorylation of Src, but had no impact on expression level of MMP-9 and phosphorylation of ERK1/2 in HCC cells. Instead, phosphorylation of AKT was significantly regulated by COL4A1. Furthermore, COL4A2, but not COL1A1 or COL3A1 also regulated phosphorylation of FAK and Src. As FAK has been reported to affect Src and AKT activation in cancer [49-52], it is convincing that 


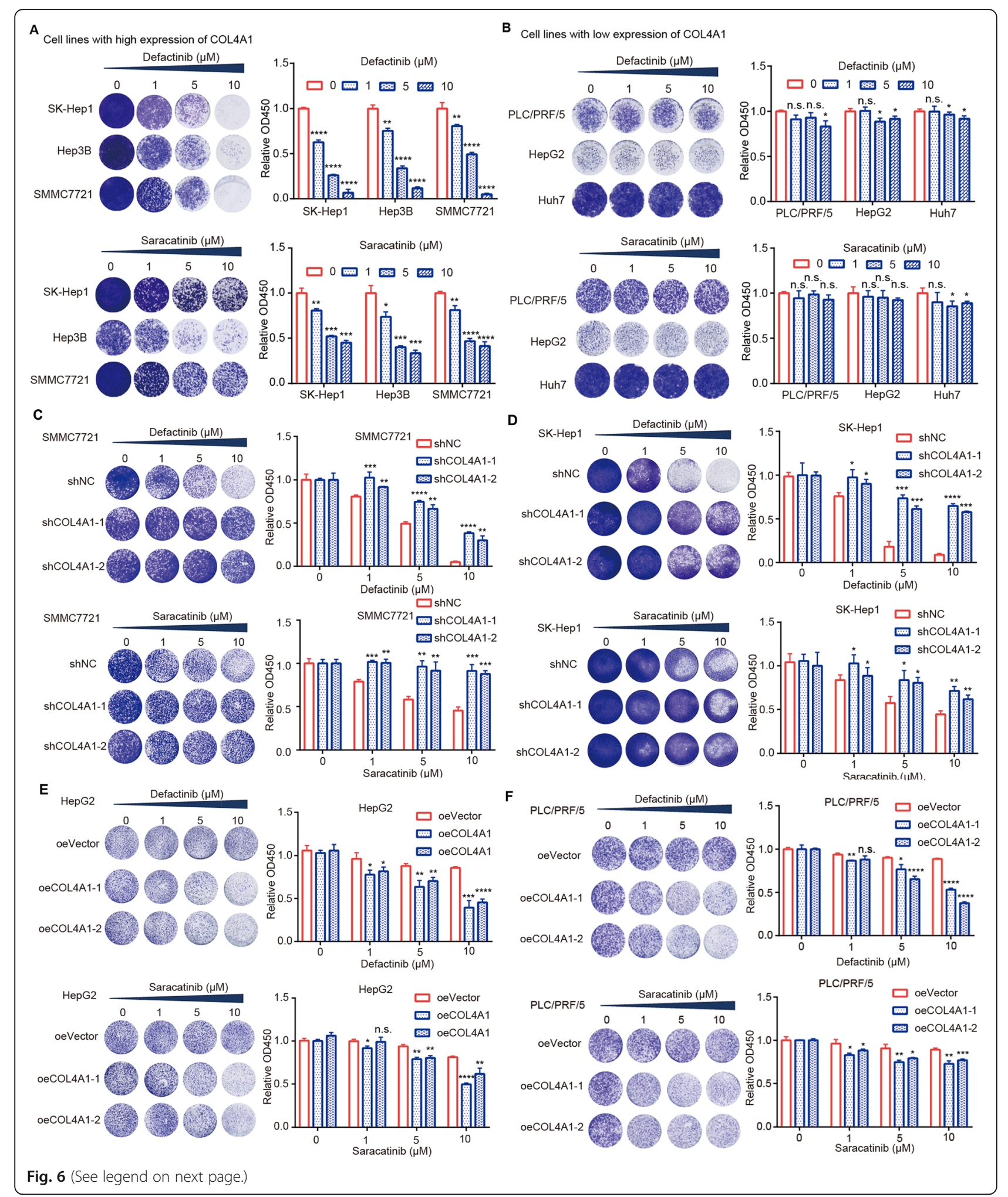


(See figure on previous page.)

Fig. 6 FAK or Src inhibitor selectively inhibits the cell viability of HCC cells with high expression of COL4A1. a\&b Cell viability was tested after treatment with inhibitors. HCC cells were treated with Defactinib (FAK inhibitor) or Saracatinib (Src inhibitor) at the indicated concentrations for $48 \mathrm{~h}$. HCC cells with high expression level of COL4A1 (a) were sensitive to Defactinib or Saracatinib treatment, but HCC cells with low expression level of COL4A1 (b) were resistance to Defactinib or Saracatinib treatment. c\&d Knockdown of COL4A1 reduced the sensitive to Defactinib or Saracatinib treatment in SMMC7721 (c) and SK-Hep1 (d). Indicated cells were treated with Defactinib (FAK inhibitor) or Saracatinib (Src inhibitor) at the indicated concentrations for $48 \mathrm{~h}$. e\&f Overexpression of COL4A1 increased the sensitive to Defactinib or Saracatinib treatment in HepG2 (e) and PLC/PRF/5 (f). Indicated cells were treated with Defactinib (FAK inhibitor) or Saracatinib (Src inhibitor) at the indicated concentrations for $48 \mathrm{~h}$. Cell viability was analyzed by crystal violet staining assay (Left) and CCK8 assay (Right), respectively. Data are presented as means \pm standard deviation. Student $t$ test, ${ }^{*} P<0.05,{ }^{* *} P<0.01,{ }^{* * *} P<0.001,{ }^{* * * *} P<0.0001$, ns, not significant

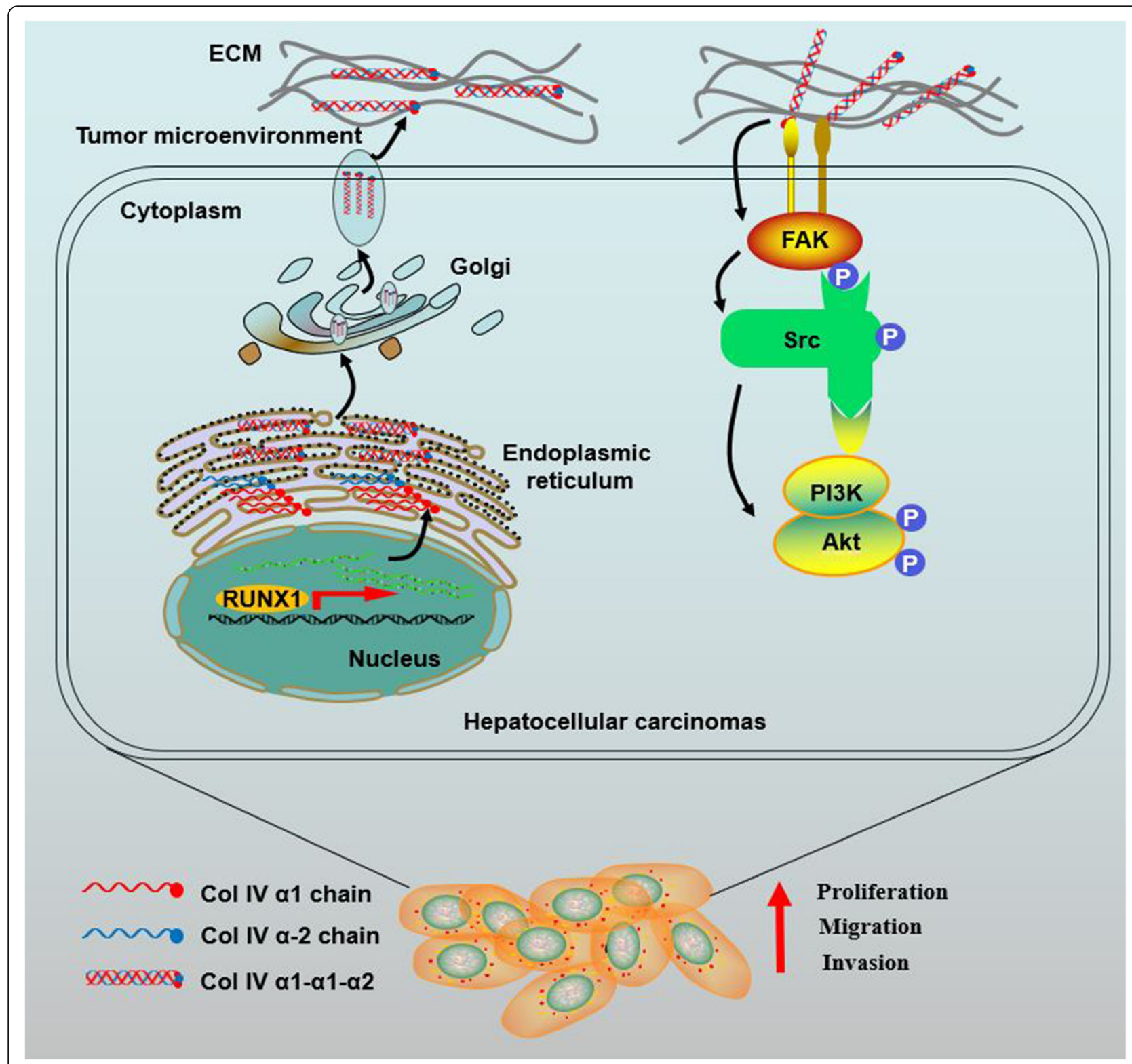

Fig. 7 Schematic diagram of COL4A1 promoting the growth and metastasis of HCC cells. COL4A1 promotes the growth, migration and invasion of HCC cells by activating FAK-Src signaling. Col IV, Collagen type IV 
COL4A1 is involved in the proliferation and migration of HCC cells through FAK-Src signaling as type IV collagen molecules.

The reason of upregulation of COL4A1 in HCC remains unclear. In this study, we demonstrated that the upregulation of COL4A1 in HCC was due to transcriptional factor RUNX1. RUNX family, including RUNX1, RUNX2 and RUNX3, are transcription factors regulating embryonic development, proliferation, differentiation and cell growth in different tissues [58]. Three RUNXs express in different tissues and regulate different target genes through binding to different coactivators or corepressors, which lead them to regulate diverse biological processes [58, 59]. In liver cancer, our results showed that RUNX1 but not RUNX2 or RUNX3 was upregulated in tumor tissue in comparison with normal tissue. More importantly, expression level of RUNX1 positively correlates with expression level of COL4A1. Overexpression of RUNX1 but not RUNX2 or RUNX3 increased the transcription of COL4A1, and knockdown of RUNX1 reduced the transcription of COL4A1. These data suggest that RUNX1 but not RUNX2 or RUNX3 is a transcriptional factor which contributes to upregulation of COL4A1 in HCC. Some studies have reported that mRNA levels of COL4A1 are also subject to posttranscriptional control such as microRNAs [60, 61], suggesting that the expression of COL4A1 in cancer may be regulated by multiple mechanisms.

How RUNX1 is upregulated in HCC remains unknown. RUNX1 is highly active in hematopoietic cell differentiation and its overexpression is necessary for tumor formation in the skin, lungs, intestines and breasts [59, 62, 63]. In epithelial ovarian cancer, RUNX1 is significantly overexpressed due to DNA hypomethylation, and could promote cancer progression [64]. In breast cancer cells, the binding of RUNXOR IncRNA trigger CpG island DNA demethylation and activate the expression of RUNX1 [65]. Chromosomal rearrangements activate the expression of RUNX1 by perturbing its transcriptional control to contribute to acute myeloid leukemia pathogenesis [66]. Whether high expression of RUNX1 in HCC through these mechanisms is worth investigating in the future. Interestingly, COL4A1 was also upregulated in breast cancer and ovarian cancer like RUNX1 (Additional file 4: Figure S1D). It is possible that overexpression of RUNX1 in these cancer types could also activate COL4A1 expression to promote tumorigenesis.

Several studies reported that COL4A1 could be a potential therapeutic target gene in head and neck squamous cell carcinoma, colorectal carcinoma, and thyroid papillary carcinoma [36]. However, the feasibility and strategy of targeting COL4A1 for HCC patients treatment is still unclear. Considering that the oncogenic function of COL4A1 in HCC relies on FAK and Src activation, we wonder whether inhibiting FAK or Src activity is an effective approach to treat HCC patients with high expression of COL4A1. Indeed, HCC cell lines with high expression of COL4A1 such as SK-Hep1, Hep3B, and SMMC7721 were more sensitive to FAK or Src inhibitor, and knockdown of COL4A1 reduced the sensitivity of those cells. In contrast, HCC cell lines with low expression of COL4A1 such as PLC/PRF/5, HepG2, and Huh7 were more resistant to FAK or Src inhibitor, and overexpression of COL4A1 increased the sensitivity. Therefore, COL4A1 may be a potential biomarker to indicate the utilization of FAK or Src inhibitor for HCC patients treatment.

\section{Conclusions}

In summary, our findings elucidate that COL4A1 functions as an oncogene to facilitate growth and metastasis in HCC via the activation of FAK-Src signaling. Upregulation of COL4A1 in HCC is due to transcriptional factor RUNX1. HCC cells with high COL4A1 expression are sensitive to the treatment with FAK or Src inhibitor (Fig. 7). Our study could help to better understand the mechanisms underlying HCC progression. COL4A1 maybe a biomarker and potential target for HCC therapy.

\section{Supplementary information}

Supplementary information accompanies this paper at https://doi.org/10. 1186/s13046-020-01650-7.

Additional file 1: Table S1. Antibodies used in this study.

Additional file 2: Table S2. Primers used in this study.

Additional file 3: Table S3. The list of differentially expressed collagen genes in HCC samples compared with normal liver tissues.

Additional file 4: Figure S1. COL4A1 is overexpressed in HCC.

Additional file 5: Figure S2. Knockdown of COL4A1 inhibits the proliferation, migration, and invasion in HepG2 cells and PLC/PRF/5 cells.

Additional file 6: Figure S3. Knockdown of COL4A2 inhibits the proliferation and migration of HCC cells.

Additional file 7: Figure S4. Knockdown of COL1A1 inhibits the proliferation and migration of HCC cells.

Additional file 8: Figure S5. Knockdown of COL3A1 has no effect on cell proliferation and migration.

Additional file 9: Figure S6. RUNX1 is a transcriptional factor of COL4A1.

Additional file 10: Figure S7. Collagen IV activates the FAK-Src signaling.

\section{Abbreviations}

HCC: Hepatocellular carcinoma; TCGA-LIHC: The Cancer Genome Atlas Liver Hepatocellular Carcinoma; Col IV: Collagen type IV; COL4A1: Collagen IV alpha1 chain; COL4A2: Collagen IV alpha2 chain; COL1A1: Collagen I alpha1 chain; COL3A1: Collagen III alpha1 chain; ECM: Extra cellular matrix; shRNACOL4A1: Small hairpin RNA expression vector targeting human COL4A1 gene; FPKM: Fragments Per Kilobase of exon model per Million mapped reads; siRNA: Small interfering RNA; SAM: CRISPR/Cas9 Synergistic Activation 
Mediator; RTCA: Real-time cell analyzer; qRT-PCR: Quantitative real-time polymerase chain reaction; NC: Negative control; ns: Not significant

\section{Acknowledgements \\ Not applicable.}

\section{Authors' contributions}

Yujun Hao, Wenxin Qin, Xiaoying Luo, and Jianren Gu designed and supervised the research. Ting Wang performed experiments, analyzed data and wrote manuscript. Yujun Hao and Wenxin Qin revised the manuscript. Jingying Hu, Huili Xu, and Haoyu Ruan cultured cells. Lin Wei and Haojie Jin finished statistical analysis. Xi Li, Fei Teng and Weihua Dong collected some HCC samples and normal liver tissues. All authors reviewed and approved the final version of the manuscript.

\section{Funding}

This work was funded by the Research Project of Shanghai Municipal Commission of Health and Family Planning $(201640261,20194$ Y0248) and the Project of State Key Laboratory of Oncogenes and Related Genes (SB19-05) to TW. This work was also supported by the Program for Professor of Special Appointment (Eastern Scholar) at Shanghai Institutions of Higher Learning (TP2017027); the Program of Shanghai Academic/Technology Research Leader (19XD1423500); and Shanghai Municipal Education CommissionGaofeng Clinical Medicine Grant Support (20171915) to YH.

\section{Availability of data and materials}

The data supporting our conclusion were obtained from the TCGA database (https://cancergenome.nih.gov), Oncomine database (https://www.oncomine. org), GEO datasets (https://www.ncbi.nlm.nih.gov/gds/), and Human Protein Atlas online database (https://www.proteinatlas.org).

\section{Ethics approval and consent to participate}

This study was approved by the Ethics Committee of the Zhongshan Hospital Biomedical Research Department and written consent was obtained from all involved patients. Animal experiments were approved by the Ethics Committee of the Renji Hospital, Shanghai Jiao Tong University School of Medicine.

\section{Consent for publication}

All authors have agreed to publish this manuscript.

\section{Competing interests}

The authors declare no conflict of interests.

\section{Author details}

'Shanghai Medical College of Fudan University, Shanghai 200032, People's Republic of China. 'State Key Laboratory of Oncogenes and Related Genes, Shanghai Cancer Institute, Renji Hospital, Shanghai Jiao Tong University School of Medicine, Shanghai 200240, People's Republic of China. ${ }^{3}$ The Fifth Affiliated Hospital of Sun Yat-sen University, Zhuhai, Guangdong 519000, People's Republic of China. ${ }^{4}$ Changzheng Hospital, Navy Medical University, Shanghai 200003, People's Republic of China.

Received: 2 April 2020 Accepted: 21 July 2020

Published online: 03 August 2020

\section{References}

1. Bray F, Ferlay J, Soerjomataram I, Siegel RL, Torre LA, Jemal A. Global cancer statistics 2018: GLOBOCAN estimates of incidence and mortality worldwide for 36 cancers in 185 countries. CA Cancer J Clin. 2018;68:394-424.

2. Cadier B, Bulsei J, Nahon P, Seror O, Laurent A, Rosa I, et al. Early detection and curative treatment of hepatocellular carcinoma: a cost-effectiveness analysis in France and in the United States. Hepatology. 2017;65:1237-48.

3. Thomas MB, Zhu AX. Hepatocellular carcinoma: the need for progress. J Clin Oncol. 2005;23:2892-9.

4. Thangavelu PU, Krenács T, Dray E, Duijf PHG. In epithelial cancers, aberrant COL17A1 promoter methylation predicts its misexpression and increased invasion. Clin Epigenetics. 2016;8:120-32.

5. Fang M, Yuan J, Peng C, Li Y. Collagen as a double-edged sword in tumor progression. Tumour Biol. 2014;35:2871-82.
6. Jarvelainen $\mathrm{H}$, Sainio A, Koulu M, Wight TN, Penttinen R. Extracellular matrix molecules: potential targets in pharmacotherapy. Pharmacol Rev. 2009;61: 198-223.

7. Alex L, Frangogiannis NG. The cellular origin of activated fibroblasts in the infarcted and remodeling myocardium. Circ Res. 2018;122:540-2.

8. Hosaki-Takamiya R, Hashimoto M, Imai Y, Nishida T, Yamada N, Mori H, et al. Collagen production of osteoblasts revealed by ultra-high voltage electron microscopy. J Bone Miner Metab. 2016;34:491-9.

9. Buchtler S, Grill A, Hofmarksrichter S, Stockert P, Schiechl-Brachner G, Rodriguez GM, et al. Cellular origin and functional relevance of collagen I production in the kidney. J Am Soc Nephrol. 2018;29:1859-73.

10. Wang H, Su Y. Collagen IV contributes to nitric oxide-induced angiogenesis of lung endothelial cells. Am J Physiol Cell Physiol. 2011;300:C979-88.

11. Abrahamson DR, Hudson BG, Stroganova L, Borza DB, St JP. Cellular origins of type IV collagen networks in developing glomeruli. J Am Soc Nephrol. 2009;20:1471-9.

12. Xu S, Xu H, Wang W, Li S, Li H, Li T, et al. The role of collagen in cancer: from bench to bedside. J Transl Med. 2019;17:309.

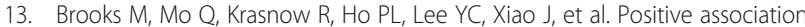
of collagen type I with non-muscle invasive bladder cancer progression. Oncotarget. 2016;7:82609-19.

14. Barcus CE, O'Leary KA, Brockman JL, Rugowski DE, Liu Y, Garcia N, et al. Elevated collagen-I augments tumor progressive signals, intravasation and metastasis of prolactin-induced estrogen receptor alpha positive mammary tumor cells. Breast Cancer Res. 2017;19:9.

15. Shintani Y, Maeda M, Chaika N, Johnson KR, Wheelock MJ. Collagen I promotes epithelial-to-mesenchymal transition in lung cancer cells via transforming growth factor-beta signaling. Am J Respir Cell Mol Biol. 2008; 38:95-104.

16. Shintani Y, Hollingsworth MA, Wheelock MJ, Johnson KR. Collagen I promotes metastasis in pancreatic cancer by activating c-Jun NH (2)terminal kinase 1 and up-regulating $\mathrm{N}$-cadherin expression. Cancer Res. 2006;66:11745-53.

17. Yang MC, Wang CJ, Liao PC, Yen CJ, Shan YS. Hepatic stellate cells secretes type I collagen to trigger epithelial mesenchymal transition of hepatoma cells. Am J Cancer Res. 2014;4:751-63.

18. Nystrom H, Naredi P, Hafstrom L, Sund M. Type IV collagen as a tumour marker for colorectal liver metastases. Eur J Surg Oncol. 2011;37:611-7.

19. Nystrom H, Tavelin B, Bjorklund M, Naredi P, Sund M. Improved tumour marker sensitivity in detecting colorectal liver metastases by combined type IV collagen and CEA measurement. Tumour Biol. 2015;36:9839-47.

20. Qiao J, Fang CY, Chen SX, Wang XQ, Cui SJ, Liu XH, et al. Stroma derived COL6A3 is a potential prognosis marker of colorectal carcinoma revealed by quantitative proteomics. Oncotarget. 2015;6:29929-46.

21. Sherman-Baust CA, Weeraratna AT, Rangel LB, Pizer ES, Cho KR, Schwartz $D R$, et al. Remodeling of the extracellular matrix through overexpression of collagen VI contributes to cisplatin resistance in ovarian cancer cells. Cancer Cell. 2003;3:377-86.

22. An JH, Lee SY, Jeon JY, Cho KG, Kim SU, Lee MA. Identification of gliotropic factors that induce human stem cell migration to malignant tumor. J Proteome Res. 2009;8:2873-81.

23. Fischer H, Salahshor S, Stenling R, Bjork J, Lindmark G, Iselius L, et al. COL11A1 in FAP polyps and in sporadic colorectal tumors. BMC Cancer. 2001;1:17.

24. Cheon DJ, Tong Y, Sim MS, Dering J, Berel D, Cui X, et al. A collagenremodeling gene signature regulated by TGF-beta signaling is associated with metastasis and poor survival in serous ovarian cancer. Clin Cancer Res. 2014:20:711-23.

25. Halsted KC, Bowen KB, Bond L, Luman SE, Jorcyk CL, Fyffe WE, et al. Collagen alpha1(XI) in normal and malignant breast tissue. Mod Pathol. 2008;21:1246-54.

26. Stelkovics E, Korom I, Marczinovits I, Molnar J, Rasky K, Raso E, et al. Collagen $\mathrm{XVII/BP180}$ protein expression in squamous cell carcinoma of the skin detected with novel monoclonal antibodies in archived tissues using tissue microarrays and digital microscopy. Appl Immunohistochem Mol Morphol. 2008;16:433-41.

27. Krenacs T, Kiszner G, Stelkovics E, Balla P, Teleki I, Nemeth I, et al. Collagen $\mathrm{XVII}$ is expressed in malignant but not in benign melanocytic tumors and it can mediate antibody induced melanoma apoptosis. Histochem Cell Biol. 2012;138:653-67.

28. Kalluri R. Basement membranes: structure, assembly and role in tumour angiogenesis. Nat Rev Cancer. 2003;3:422-33. 
29. Karsdal MA, Nielsen SH, Leeming DJ, Langholm LL, Nielsen MJ, ManonJensen $T$, et al. The good and the bad collagens of fibrosis - their role in signaling and organ function. Adv Drug Deliv Rev. 2017;121:43-56.

30. Li T, Leng XS, Zhu JY, Wang G. Suppression of hedgehog signaling regulates hepatic stellate cell activation and collagen secretion. Int J Clin Exp Pathol. 2015:8:14574-9.

31. Kuo DS, Labelle-Dumais C, Gould DB. COL4A1 and COL4A2 mutations and disease: insights into pathogenic mechanisms and potential therapeutic targets. Hum Mol Genet. 2012;21:R97-110.

32. Gould DB, Phalan FC, Breedveld GJ, van Mil SE, Smith RS, Schimenti JC, et al. Mutations in Col4a1 cause perinatal cerebral hemorrhage and porencephaly. Science. 2005;308:1167-71.

33. Plaisier E, Gribouval O, Alamowitch S, Mougenot B, Prost C, Verpont MC, et al. COL4A1 mutations and hereditary angiopathy, nephropathy, aneurysms, and muscle cramps. N Engl J Med. 2007:357:2687-95.

34. Labelle-Dumais C, Dilworth DJ, Harrington EP, de Leau M, Lyons D, Kabaeva Z, et al. COL4A1 mutations cause ocular dysgenesis, neuronal localization defects, and myopathy in mice and Walker-Warburg syndrome in humans. PLoS Genet. 2011;7:e1002062.

35. Miyake M, Hori S, Morizawa Y, Tatsumi Y, Toritsuka M, Ohnishi S, et al. Collagen type IV alpha 1 (COL4A1) and collagen type XIII alpha 1 (COL13A1) produced in cancer cells promote tumor budding at the invasion front in human urothelial carcinoma of the bladder. Oncotarget. 2017;8:36099.

36. Jin R, Shen J, Zhang T, Liu Q, Liao C, Ma H, et al. The highly expressed COL4A1 genes contributes to the proliferation and migration of the invasive ductal carcinomas. Oncotarget. 2017;8:58172-83.

37. Jin H, Wang C, Jin G, Ruan H, Gu D, Wei L, et al. Regulator of Calcineurin 1 gene isoform 4, Down-regulated in hepatocellular carcinoma, prevents proliferation, migration, and invasive activity of Cancer cells and metastasis of Orthotopic tumors by inhibiting nuclear translocation of NFAT1. Gastroenterology. 2017;153:799-811.

38. Stephens DJ. Cell biology: collagen secretion explained. Nature. 2012;482:474-5.

39. Pollner R, Schmidt C, Fischer G, Kuhn K, Poschl E. Cooperative and competitive interactions of regulatory elements are involved in the control of divergent transcription of human Col4A1 and Col4A2 genes. FEBS Lett. 1997:405:31-6.

40. Ma HP, Chang HL, Bamodu OA, Yadav VK, Huang TY, Wu A, et al. Collagen 1A1 (COL1A1) Is a Reliable Biomarker and Putative Therapeutic Target for Hepatocellular Carcinogenesis and Metastasis. Cancers (Basel). 2019;11:768800.

41. Zheng X, Liu W, Xiang J, Liu P, Ke M, Wang B, et al. Collagen I promotes hepatocellular carcinoma cell proliferation by regulating integrin beta1/FAK signaling pathway in nonalcoholic fatty liver. Oncotarget. 2017;8:95586-95.

42. Zheng Q, Zhou G, Morello R, Chen Y, Garcia-Rojas X, Lee B. Type X collagen gene regulation by Runx2 contributes directly to its hypertrophic chondrocyte-specific expression in vivo. J Cell Biol. 2003;162:833-42.

43. Higashikawa A, Saito T, Ikeda T, Kamekura S, Kawamura N, Kan A, et al. Identification of the core element responsive to runt-related transcription factor 2 in the promoter of human type X collagen gene. Arthritis Rheum. 2009;60:166-78

44. Wei J, Shimazu J, Makinistoglu MP, Maurizi A, Kajimura D, Zong H, et al. Glucose uptake and Runx2 synergize to orchestrate osteoblast differentiation and bone formation. Cell. 2015;161:1576-91.

45. Khoshnoodi J, Pedchenko V, Hudson BG. Mammalian collagen IV. Microsc Res Tech. 2008;71:357-70.

46. Chen SY, Lin JS, Yang BC. Modulation of tumor cell stiffness and migration by type IV collagen through direct activation of integrin signaling pathway. Arch Biochem Biophys. 2014:555-556:1-8.

47. Hodgson L, Henderson AJ, Dong C. Melanoma cell migration to type IV collagen requires activation of NF-kappaB. Oncogene. 2003:22:98-108.

48. Macias-Perez I, Borza C, Chen X, Yan X, Ibanez R, Mernaugh G, et al. Loss of integrin alpha1beta1 ameliorates Kras-induced lung cancer. Cancer Res. 2008;68:6127-35.

49. Kim SH, Turnbull J, Guimond S. Extracellular matrix and cell signalling: the dynamic cooperation of integrin, proteoglycan and growth factor receptor. J Endocrinol. 2011;209:139-51.

50. Bolos V, Gasent JM, Lopez-Tarruella S, Grande E. The dual kinase complex FAK-Src as a promising therapeutic target in cancer. Onco Targets Ther. 2010;3:83-97.

51. Mitra SK, Schlaepfer DD. Integrin-regulated FAK-Src signaling in normal and cancer cells. Curr Opin Cell Biol. 2006:18:516-23.
52. Mitra SK, Hanson DA, Schlaepfer DD. Focal adhesion kinase: in command and control of cell motility. Nat Rev Mol Cell Biol. 2005;6:56-68.

53. Espinosa NR, Salazar EP. Native type IV collagen induces an epithelial to mesenchymal transition-like process in mammary epithelial cells MCF10A. Int J Biochem Cell Biol. 2012;44:2194-203.

54. Cortes-Reynosa P, Robledo T, Macias-Silva M, Wu SV, Salazar EP. Src kinase regulates metalloproteinase- 9 secretion induced by type IV collagen in MCF-7 human breast cancer cells. Matrix Biol. 2008;27:220-31.

55. Papaioannou I, Xu S, Denton CP, Abraham DJ, Ponticos M. STAT3 controls COL1A2 enhancer activation cooperatively with JunB, regulates type I collagen synthesis posttranscriptionally, and is essential for lung myofibroblast differentiation. Mol Biol Cell. 2018;29:84-95.

56. Liu Y, Zhang J, Chen Y, Sohel H, Ke X, Chen J, et al. The correlation and role analysis of COL4A1 and COL4A2 in hepatocarcinogenesis. Aging (Albany NY). 2020;12:204-23.

57. Burnier JV, Wang N, Michel RP, Hassanain M, Li S, Lu Y, et al. Type IV collagen-initiated signals provide survival and growth cues required for liver metastasis. Oncogene. 2011;30:3766-83.

58. Otalora-Otalora BA, Henriquez B, Lopez-Kleine L, Rojas A. RUNX family: oncogenes or tumor suppressors (review). Oncol Rep. 2019;42:3-19.

59. Yamagata T, Maki K, Mitani K. Runx1/AML1 in normal and abnormal hematopoiesis. Int J Hematol. 2005;82:1-8.

60. Sengupta S, den Boon JA, Chen IH, Newton MA, Stanhope SA, Cheng YJ, et al. MicroRNA 29c is down-regulated in nasopharyngeal carcinomas, upregulating mRNAs encoding extracellular matrix proteins. Proc Natl Acad Sci U S A. 2008;105:5874-8.

61. Du B, Ma LM, Huang MB, Zhou H, Huang HL, Shao P, et al. High glucose down-regulates miR-29a to increase collagen IV production in HK-2 cells. FEBS Lett. 2010;584:811-6.

62. Taniuchi I, Osato M, Ito Y. Runx1: no longer just for leukemia. EMBO J. 2012; 31:4098-9.

63. Scheitz CJ, Lee TS, McDermitt DJ, Tumbar T. Defining a tissue stem celldriven Runx1/Stat3 signalling axis in epithelial cancer. EMBO J. 2012;31: 4124-39.

64. Keita M, Bachvarova M, Morin C, Plante M, Gregoire J, Renaud MC, et al. The RUNX1 transcription factor is expressed in serous epithelial ovarian carcinoma and contributes to cell proliferation, migration and invasion. Cell Cycle. 2013;12:972-86.

65. Nie $Y$, Zhou L, Wang H, Chen N, Jia L, Wang C, et al. Profiling the epigenetic interplay of IncRNA RUNXOR and oncogenic RUNX1 in breast cancer cells by gene in situ cis-activation. Am J Cancer Res. 2019;9:1635-49.

66. Cheng CK, Wong T, Wan T, Wang AZ, Chan N, Chan N, et al. RUNX1 upregulation via disruption of long-range transcriptional control by a novel t $(5 ; 21)(q 13 ; q 22)$ translocation in acute myeloid leukemia. Mol Cancer. 2018; $17: 133$.

\section{Publisher's Note}

Springer Nature remains neutral with regard to jurisdictional claims in published maps and institutional affiliations.

Ready to submit your research? Choose BMC and benefit from:

- fast, convenient online submission

- thorough peer review by experienced researchers in your field

- rapid publication on acceptance

- support for research data, including large and complex data types

- gold Open Access which fosters wider collaboration and increased citations

- maximum visibility for your research: over $100 \mathrm{M}$ website views per year

At $\mathrm{BMC}$, research is always in progress.

Learn more biomedcentral.com/submission 